\title{
Including autapomorphies is important for paleontological tip- dating with clocklike data, but not with non-clock data
}

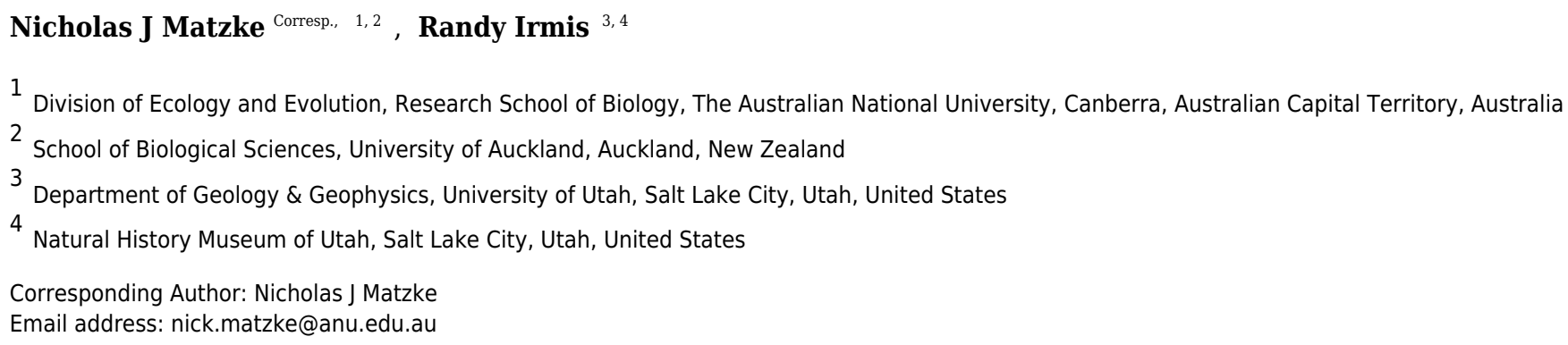

Tip-dating, where fossils are included as dated terminal taxa in Bayesian dating inference, is an increasingly popular method. Data for these studies often come from morphological character matrices originally developed for non-dated, and usually parsimony, analyses. In parsimony, only shared derived characters (synapomorphies) provide grouping information, so many character matrices have an ascertainment bias: they leave out autapomorphies (unique derived character states), which are considered uninformative. There has been no study of the affect of this ascertainment bias in tip-dating, but autapomorphies can be informative in model-based inference. We expected that excluding autapomorphies would shorten the morphological branchlengths of terminal branches, and thus bias downwards the time branchlengths inferred in tip-dating. We tested for this effect using a matrix for Carboniferous-Permian eureptiles where all autapomorphies had been deliberately coded. Surprisingly, date estimates are virtually unchanged when autapomorphies are excluded, although we find large changes in morphological rate estimates and small effects on topological and dating confidence. We hypothesized that the puzzling lack of effect on dating was caused by the non-clock nature of the eureptile data. We confirm this explanation by simulating strict clock and non-clock datasets, showing that autapomorphy exclusion biases dating only for the clocklike case. A theoretical solution to ascertainment bias is computing the ascertainment bias correction $\left(M k_{\text {parsinf }}\right)$, but we explore this correction in detail, and show that it is computationally impractical for typical datasets with many character states and taxa. Therefore we recommend that palaeontologists collect autapomorphies whenever possible when assembling character matrices. 
1 Title: Including autapomorphies is important for paleontological tip-dating with clocklike

2 data, but not with non-clock data

3

4 Matzke, Nicholas J. ${ }^{1,2^{*}}$; Irmis, Randall B..$^{3,4}$

5

$6{ }^{1}$ Division of Ecology and Evolution, Research School of Biology, The Australian National

7 University, Canberra, Australian Capital Territory, Australia

$8{ }^{2}$ School of Biological Sciences, University of Auckland, Auckland, New Zealand

$9{ }^{3}$ Department of Geology \& Geophysics, University of Utah, Salt Lake City, Utah, United States

$10{ }^{4}$ Natural History Museum of Utah, Salt Lake City, Utah, United States

$11{ }^{*}$ Corresponding author. Email: nick.matzke@anu.edu.au

12

\section{Abstract}

14 Tip-dating, where fossils are included as dated terminal taxa in Bayesian dating inference, is an increasingly popular method. Data for these studies often come from morphological character matrices originally developed for non-dated, and usually parsimony, analyses. In parsimony,

17 only shared derived characters (synapomorphies) provide grouping information, so many character matrices have an ascertainment bias: they leave out autapomorphies (unique derived

19 character states), which are considered uninformative. There has been no study of the affect of

20 this ascertainment bias in tip-dating, but autapomorphies can be informative in model-based

21 inference. We expected that excluding autapomorphies would shorten the morphological

22 branchlengths of terminal branches, and thus cause artificially shortened time branchlengths 
23 inferred in tip-dating. We tested for this effect using a matrix for Carboniferous-Permian

24 eureptiles where all autapomorphies had been deliberately coded. Surprisingly, date estimates

25 are virtually unchanged when autapomorphies are excluded, although we find large changes in

26 morphological rate estimates and small effects on topological and dating confidence. We

27 hypothesized that the puzzling lack of effect on dating was caused by the non-clock nature of

28 the eureptile data. We confirm this explanation by simulating strict clock and non-clock

29 datasets, showing that autapomorphy exclusion biases dating only for the clocklike case. A

30 theoretical solution to ascertainment bias is computing the ascertainment bias correction

$31\left(\mathrm{M} k_{\text {parsinf }}\right)$, but we explore this correction in detail, and show that it is computationally

32 impractical for typical datasets with many character states and taxa. Therefore we recommend

33 that palaeontologists collect autapomorphies whenever possible when assembling character

34 matrices.

35

36

37

38 


\section{Introduction}

In parsimony phylogenetic analyses, the only data informative for reconstructing the tree topology are those with grouping information: potentially shared, derived character states (synapomorphies; Hennig et al. 1999). An autapomorphy - a state unique to one terminal taxon or Operational Taxonomic Unit (OTU; Mishler 2005) -- contributes one step to any possible topology. Therefore, autapomorphies are routinely excluded from further analysis in cladistics programs (e.g., the TNT xinact and info commands (Goloboff et al. 2008); the PAUP* exclude command (Swofford 2003); and see Yeates 1992), and autapomorphic characters are often not even collected during assembly of a character-taxon matrix.

In model-based inference, autapomorphies can be informative (Lewis 2001; Wright \& Hillis 2014), because autapomorphies contribute information about the overall rate of change in the character matrix and site-specific rate heterogenetity. An insufficiently recognized point is that autapomorphies might be particularly important in "tip-dating" analyses, where terminal taxa include fossils with ages older than the present day (Alexandrou et al. 2013; Pyron 2011; Ronquist et al. 2012; Wood et al. 2013). Tip-dating analyses might be expected to be particularly sensitive to autapomorphies: all autapomorphies occur on terminal branches by definition, so their exclusion will shorten the morphological branchlengths of terminal branches (and thus presumably their time branchlengths), and perhaps increase estimated branch-wise rate variation. 
61 An alternative to inclusion of autapomorphies is ascertainment-bias correction, where the

62 likelihood of unobservable character patterns, $L_{\text {unobs, }}$ is calculated, and the likelihood of the

63 observed data is normalized by dividing by $1-L_{\text {unobs }}$ (Felsenstein 1992; Lewis 2001). The two

64 common corrections are the Markov-k model with an ascertainment bias correction for the

65 unobservability of invariant characters (Mk-variable-only, or Mkv; Lewis 2001), and Markov-k

66 with an ascertainment bias correction for parsimony-uninformative characters, $\mathrm{M} k_{\text {parsinf }}$ (Allman

67 et al. 2010; Ronquist \& Huelsenbeck 2003). These corrections are options in MrBayes and can

68 be implemented in Beast1/Beast2 XML, but several studies briefly mention that the scalability

69 and correctness of $\mathrm{M} k_{\text {parsinf }}$ computations may be problematic (dos Reis et al. 2016; Koch \&

70 Holder 2012; Matzke 2016).

71

72 The effect inclusion/exclusion of autapomorphies and ascertainment-bias correction has not

73 been studied in a tip-dating context. Datasets appropriate for doing so are rare because they

74 need to systematically collect all autapomorphies, as well as dates for the OTUs. Müller \& Reisz

75 (2006) constructed an all-fossil, morphological matrix of early eureptiles and tested the effect

76 of inclusion/exclusion of autapomorphies in undated Bayesian inference, and recommended

77 including autapomorphies. Lee and Palci (2015) discussed the importance of autapomorphies

78 for tip-dating, but did test the effect of their inclusion/exclusion. We obtained dates for Müller

79 and Reisz's taxa, and use the dataset to test the effects of autapomorphy inclusion. Surprisingly,

80 no effect on dates was found. This might be due to the non-clocklike nature of the dataset, an

81 explanation we confirm with a simulation study that shows autapomorphy exclusion biases

82 terminal branchlength estimates when the data are highly clocklike, but not in a non-clock 
83 dataset. We also examine the $\mathrm{M} k_{\text {parsinf }}$ correction and show that it scales poorly for characters

84 with more than two states, limiting its usability.

85

86

Methods

87 Data. The morphological matrix was taken from Müller and Reisz (2006). The date ranges for

88 OTUs were derived from the literature, following best practices guidelines (2012). Correlation

89 between time and morphological branchlengths in a TNT parsimony analysis was used as a

90 rough assessment of clocklike behavior (for further description of all methods, as well as all

91 data and scripts used, see Supplemental Material).

92

93 Tip-dating eureptiles. Tip-dating in Beast2 (Bouckaert et al. 2014; Drummond \& Bouckaert

94 2015) with Birth-Death-Serial Sampling (BDSS) or SA-BDSS (Sampled Ancestors) tree models

95 (Gavryushkina et al. 2015; Gavryushkina et al. 2014) requires a specialized XML input file. To set

96 this up, we used BEASTmasteR (Alexandrou et al. 2013; Matzke 2015; Matzke \& Wright 2016), a

97 set of R functions that convert NEXUS character matrices, an Excel file containing tip date

98 ranges, and other priors and settings, into XML. Three different site models were used: Mk,

$99 \mathrm{Mkv}$, and $\mathrm{M} k_{\text {parsinf. }}$ The summary Maximum Clade Credibility (MCC) trees were plotted with $95 \%$

100 highest posterior densities (HPDs) on inferred node (blue) and tip dates (red) using

101 BEASTmasteR functions and custom R scripts. Mean node dates, node 95\% HPD widths,

102 posterior probabilities, and rates were compared between pairs of analyses (with/without

103 autapomorphies) for nodes/bipartitions shared between analyses $(n=14)$, with the Wilcoxon 
104 signed-rank test (WSRT) for paired samples. Due to the small number of tests, no multiple-test 105 correction was used.

106

107 Simulation. To test whether clocklike behavior is needed to observe effects of autapomorphy

108 exclusion on date estimates, a BDSS tree similar in size to the empirical dataset (30 species) was

109 simulated using TreeSim (Stadler 2015). A "strict clock" dataset of 1000 binary characters was

110 simulated on this tree under the $\mathrm{Mk}$ model with a rate low enough (0.05) that a substantial

111 proportion of the characters (577/1000) were invariant or autapomorphic. A "non-clock"

112 dataset was produced by reshuffling the time-branchlengths of the simulated tree, and then

113 simulating another 1000 characters at the same rate. Datasets were filtered to produce

114 variable-only and parsimony-informative-only datasets, effectively imposing ascertainment

115 bias. Beast2 runs were conducted on both simulated datasets under $\mathrm{Mk}, \mathrm{Mkv}$, and $\mathrm{M} k_{\text {parsinf }}$

116 using the same setup as for the empirical analysis. All scripts, Beast2 inputs and outputs, and

117 further details of the analyses are available in Supplemental Material (SM).

118

119 Scalability of the $M \mathrm{k}_{\text {parsinf }}$ correction. Although listed as an option in MrBayes for a over a

120 decade, surprisingly, $\mathrm{M} k_{\text {parsinf }}$ has not been formally described anywhere in the literature,

121 leading to widespread lack of knowledge of how it works and whether or not it is

122 computationally feasible on typical datasets. Nor has there been any formal treatment of its

123 computational scalability. The key issue is the number of unobservable character patterns for a

124 character with a particular number of states, as the likelihood of each unobservable pattern

125 must be calculated. While this is feasible for a binary character (which appears to be the 
126 assumption made by MrBayes), for a dataset with many taxa and multistate characters, the

127 number of unobservable site patterns rapidly climbs into the millions. Appendix 1 contains a

128 derivation of the number of likelihood calculations required by $\mathrm{M} k_{\text {parsinf, }}$ and discussion of

129 computational scalability.

130

131 Results

132 Tip-dating eureptiles. Fourteen bipartitions were shared by the summary trees of all analyses.

133 MCC trees for two runs are illustrated in Figure 1; for all runs, see Supplemental Figure 1.

134 Summary statistics of key parameters are shown in Table 1. Linear regression of tip age against

135 the root-to-tip distance in a parsimony analysis (the number of morphological steps on all

136 branches leading to a tip, see a similar approach for molecular data by Rambaut et al. 2016)

137 indicated that time and parsimony branchlengths were not correlated. This is evidence that the

138 morphological characters in the eureptile dataset are not evolving in a clocklike manner.

139

140

Inferred node dates. Estimates of the root age are almost identical between analyses with and

141 without autapomorphies (Table 1). Comparing mean dates for nodes shared across the MCC

142 trees yields no significant differences (WTST, two-sided, $n=14$ shared nodes), with $P=0.359$ for

143 the $M k$ inference, and $P=0.280$ for $M k v$ inferences.

145 Dating uncertainty (HPD widths). Adding data should reduce uncertainty, especially with small

146 morphological datasets. The null hypothesis, that the no-autapomorphies dataset does not

147 have greater HPD widths, was rejected for the Mk inferences (including vs. excluding 
148 autapomorphies, 9.20 vs. 9.94, $P=0.023$, one-sided WSRT); the result for the Mkv inferences

149 was only suggestive (9.37 vs. $9.66, P=0.105)$.

150

151 Posterior probabilities (PPS). PPs were higher for runs including autapomorphies under both the

152 Mk model (including vs. excluding autapomorphies, 0.902 vs. 0.756$)$ and the Mkv model (0.900

153 vs. 0.835). The null hypothesis, that the no-autapomorphies dataset does not have smaller PPs,

154 was rejected at a significance level of 0.05 for both the $M k$ inference $(P=0.0095$, one-sided

155 WSRT) and Mkv inference $(P=0.0252)$.

156

157 Relaxed clock. The mean of the relaxed clock rate is dramatically affected by inclusion of

158 autapomorphies, under both the $\mathrm{M} k$ model (with autapomorphies, rate mean $=0.0782$ changes

159 per site per million years, 95\% HPD=[0.015, 0.159]; without: 0.788 [0.0305, 3.982]) and the Mkv

160 model (with: 0.0376 [0.0074, 0.0840]; without: 0.550 [0.0228, 2.655]) (tests in SM), roughly a

161 increase of an order of magnitude in both cases. The $\mathrm{M} k_{\text {parsinf }}$ run of the no-autapomorphies

162 dataset yielded an intermediate clock rate $(0.235,95 \%$ HPD $=[0.0142,0.664])$.

163

164 Simulations. Figure 2 shows the simulation procedure and key comparisons. Similar tree

165 topologies were inferred under all datasets, but estimated time-branchlengths differed. When

166 the characters are clocklike and autapomorphies are included, inferred time-branchlengths are

167 highly accurate (2B). However, when autapomorphies are excluded, inferred terminal

168 branchlengths are biased downwards, and accuracy decreases for all branchlengths. The effect 
169 in $2 \mathrm{C}$ can also be seen by comparing inference while including vs. excluding autapomorphies,

170 when the characters are clocklike (2D), but this effect disappears for non-clock data (2E).

171

172 Feasibility of $M \mathrm{k}_{\text {parsinf. }}$ Equations in Appendix 1 demonstrate that $M \mathrm{k}_{\text {parsinf }}$ can be feasible for 2-

173 state characters, and for 3-state characters on small datasets ( 10 times slower for our

174 dataset), but rapidly becomes computationally impractical as the number of taxa or states

175 increases. The number of unobservable site patterns for various combinations of numbers of

176 taxa and character states are shown in Table 2.

177

\section{Discussion}

179 Although estimated mean rate parameters for the eureptile dataset dropped dramatically (by

18010 times or more) when autapomorphies were included (and somewhat less when

181 ascertainment-bias correction was used instead), the downstream effects on confidence were

182 small (Table 1; SM), and there was no detectable effect on date inference. This seems

183 surprising, because the exclusion of autapomorphies must reduce the number of morphological

184 changes on terminal branches. However, reflection on the interaction between non-clocklike

185 data, and the flexibility of relaxed-clock Bayesian tip-dating methods, provides an explanation.

186 If the character data are non-clocklike, then the method will estimate a high rate of branchwise

187 rate variation, indicating lack of correlation between time elapsed and morphological

188 branchlength. In this situation, most of the dating information for the analysis comes from the

189 serial-sampling of fossil tips rather than morphological branchlengths. If morphological 
190 branchlength is not correlated with time, this remains true whether or not autapomorphies are

191 included, and adding autapomorphies is not likely to change the dating inference.

192

193 Our simulation results (Figure 2) confirm this explanation. The analysis of the empirical

194 eureptile dataset is likely similar to the situation shown in Figure 2E: inferred time

195 branchlengths are roughly the same whether or not autapomorphies are included. However, on

196 a clocklike dataset, exclusion of autapomorphies clearly has an effect (Figure 2C). This suggests

197 that the importance of including autapomorphies in tip-dating analyses depends on whether or

198 not the characters have clocklike behavior. Unfortunately, assessing clocklike behavior will be

199 more difficult when autapomorphies have been ignored or gathered only inconsistently (as is

200 common).

201

202 An alternative to coding autapomorphies is the $\mathrm{M} k_{\text {parsinf }}$ model. However, Appendix 1 shows

203 that it scales too poorly to be generally useful for characters with large number of states (Table

204 2; SM). All versions of MrBayes back to at least 3.1.2 allow a "coding=informative"

205 ascertainment bias correction to be specified, but the increase in computation time for a run

206 with a single discrete character is very similar whether the character has 2, 3, 4, or 5 states

207 (tested on MrBayes versions 3.1.2 through 3.2.6, and the 3.2.7 development version). This

208 suggests that $\mathrm{M} k_{\text {parsinf }}$ may be implemented assuming only binary characters, and may be

209 formally incorrect for multistate characters (as briefly noted by dos Reis et al. 2016; Matzke

210 2016), despite many usages in the literature. However, as most morphological datasets are 
211 dominated by binary characters, this issue may have limited impact on inference, and requires

212 further study.

213

214 Conclusion

215 Our study indicates that the common practice of repurposing character matrices devised for

216 parsimony and undated Bayesian analyses may not be sufficient in the world of Bayesian tip-

217 dating. For higher quality datasets (many characters, clocklike behavior), the bias in dating

218 introduced by ignoring autapomorphies may become significant. Additionally, ascertainment

219 bias corrections are at present computationally impractical for many datasets with multistate

220 characters. Finally, autapomorphies have additional utility for improving estimates of rates and

221 rate variation, for species identification, for measuring disparity, and because autapomorphies

222 may become synapomorphies when new taxa are described. Therefore, we recommend that

223 autapomorphies be coded and used whenever possible.

224

225 Appendix 1: Derivation of the $\mathbf{M} \boldsymbol{k}_{\text {parsinf }}$ ascertainment bias correction, and problems with

226 scalability

227

228 One potential alternative to our recommendation to code autapomorphies could be to employ

229 the Mk-Parsimony-Informative model ( $\left.\mathrm{M} k_{\text {parsinf }}\right)$, that is, the Markov- $k$ model with an

230 ascertainment bias correction for not just the unobservability of invariant characters, but also

231 for the unobservability of autapomorphic characters (in a dataset that excludes autapomorphic

232 characters). 
234 A significant question is whether or not the $M k_{\text {parsinf }}$ model can actually be employed on typical 235 datasets. MrBayes, since at least version 3.1.2, does allow the $\mathrm{M} k_{\text {parsinf }}$ ascertainment bias 236 correction as an option ("Iset coding=informative"; (Ronquist et al. 2011), p. 146, or

237 http://mrbayes.sourceforge.net/wiki/index.php/Evolutionary Models Implemented in MrBay

238 es 3\#Standard Discrete .28Morphology.29 Model), but does not contain an extensive

239 description of how it works, and the model does not seem to have been formally described in

240 any publication. Allman et al. (Allman et al. 2010) analyse model identifiability in the $\mathrm{M} k_{\text {parsinf }}$

241 context, but only cite Nylander et al. (Nylander et al. 2004) for the model; Nylander et al. in turn

242 cite Ronquist and Huelsenbeck, "in prep.," which appears to be an uncorrected reference to 243 their cited (Ronquist \& Huelsenbeck 2003), the MrBayes 3 publication.

244

245 There is therefore a need to explore $\mathrm{M} k_{\text {parsinf }}$ in detail. Ascertainment bias correction works by

246 enumerating site patterns that are unobservable, calculating their likelihood under the model,

247 and then re-normalizing the observed data likelihood at each site by dividing by $1-L$, where $L$ is

248 the likelihood of the unobservable site pattern(s). In Felsenstein (Felsenstein 1992), the

249 unobservable pattern was "no restriction site observed," i.e., a column of all Os. In the Mkv

250 model, the unobservable patterns include a column of all 0 s, a column of all $1 \mathrm{~s}$, etc., up to the

251 number of character states, $n$, in a particular character.

252

253 However, the situation becomes much more complicated for the $\mathrm{M} k_{\text {parsinf }}$ ascertainment bias

254 correction (Supplemental Table 1). The difficulty (mentioned briefly in (dos Reis et al. 2016; 
255 Matzke 2016) is that the number of unobservable site patterns scales very poorly with number

256 of character states and number of taxa. For example, for a 100 taxon data matrix and a 3-state

257 character, the following is an unobservable site pattern: a column that consists of all 0s, a single

2581 for taxon 99, and a single 2 for taxon 100. But any other variant of this pattern is also

259 unobservable: all 0s, except state 1 at position 1, and state 2 at position 2, etc. Additional

260 unobservable patterns include all 1s except for two taxa and all 2 s except for two taxa. Also

261 unobservable are all patterns that are invariant (all 0s, all 1s, all 2s), and all patterns that are

262 invariant except for one taxon.

263

264 Formally speaking, if $n$ is the number of taxa, and $k$ is the number of states in a character, then

265 there are $k^{n}$ possible patterns for that character. We can calculate the number of site patterns

266 that are unobservable under $M k_{\text {parsinf }}$ by first selecting the number of character states, $i$, found

267 in a particular unobservable site pattern. For example, in a character assumed to have $k=5$

268 states, the number of states found in a particular pattern could be $i=1$ (i.e., an invariant site),

$269 i=2, \ldots, i=5$. For each $i$, there are

$$
\left(\begin{array}{l}
k \\
i
\end{array}\right)
$$

270 ways to pick which of the $k$ character states will be found in found in the site pattern.

271 Conditional on $i$ character states in a particular unobservable site pattern, one of them will be

272 the "dominant" state (held by all taxa, except for the autapomorphic taxa), and ( $i-1)$ of the

273 character states will be autapomorphies. There are 


$$
\left(\begin{array}{l}
i \\
1
\end{array}\right)
$$

274 ways to choose which character state is dominant. Conditional on the dominant character

275 state, there are

$$
\left(\begin{array}{c}
n \\
i-1
\end{array}\right)
$$

276 ways to choose which taxa will be autapomorphic. Conditional on which taxa are

277 autapomorphic, there are ${ }_{i-1} P_{i-1}$ permutations of ways to assign the $(i-1)$ character states to 278 the $(i-1)$ autapomorphic taxa. This is calculated

279

$$
\frac{(i-1) !}{((i-1)-(i-1)) !}=\frac{(i-1) !}{0 !}=(i-1) !
$$

281 Taking the product of equations 1-4 and summing over all $i$ yields

$$
\begin{gathered}
\sum_{i=1}^{k}\left(\begin{array}{l}
k \\
i
\end{array}\right)\left(\begin{array}{c}
i \\
1
\end{array}\right)\left(\begin{array}{c}
n \\
i-1
\end{array}\right)(i-1) ! \\
\sum_{i=1}^{k}\left(\begin{array}{l}
k \\
i
\end{array}\right) i !\left(\begin{array}{c}
n \\
i-1
\end{array}\right)
\end{gathered}
$$

282 autapomorphic patterns that are unobservable under the $\mathrm{M} k_{\text {parsinf }}$ ascertainment bias

283 correction, for a character with $k$ states. This equation is implemented in the R function

284 num_unobservable_patterns_ParsInf, included in the Supplemental Material (and available

285 online via GitHub Gist, at: https://gist.github.com/nmatzke/8f80723b6e1fc80ed5ac ).

286 
287 Calculating the number of unobservable patterns for a range of numbers of taxa and states

288 (Supplemental Table 1) shows that, for a 100-taxon morphological matrix, the presence of 289 characters with 3 states in the matrix will necessitate calculating the likelihood for 30,303 290 additional site patterns. This is computationally imaginable, although it will substantially slow

291 the MCMC search for a morphological dataset, which usually has only a few hundred 292 characters. The presence of a 4-state character requires 4,000,804 unobservable patterns. For 293 a 6-state character there are over 57 billion.

In Beast2, unobservable site patterns have to be physically listed in the XML input; even with a script to write out the patterns, users can certainly imagine the difficulty of saving and manipulating XML files containing millions of unobservable patterns. Inspection of the MrBayes code seems to indicate that the $\mathrm{M} k_{\text {parsinf }}$ correction assumes binary characters only (which is computationally feasible; Supplemental Table 1); but this leaves open the question of what calculation, is being done on characters with more than two states.

This is problematic, as many researchers (e.g. Dembo et al. 2016) are probably under the impression that $\mathrm{M} k_{\text {parsinf }}$ ascertainment bias correction works for any number of character states. It is possible that this issue is of little significance. After all, most morphological 305 characters are binary. Also, as the number of taxa and character states increases, the fraction of the total number of possible patterns $\left(k^{n}\right)$ that are unobservable (equation 5$)$ decreases

307 precipitously (Supplemental Tables 2, 3). Thus, perhaps the likelihood of unobservable sites 
309 (Ronquist et al. 2011), pp. 146-147), where the authors state they observed that as the total

310 tree length (sum of branchlengths in terms of number of expected changes per site) increases

311 e.g. above 20-30 taxa, the ascertainment bias correction becomes negligible. However, this

312 may depend greatly on the "true" rates - if they are low, and thus invariant

313 and autapomorphic patterns are some of the most probable patterns, then the likelihood

314 correction from unobservable patterns could be large. As this paper showed, in the case of the

315 25-taxon eureptile dataset, just switching from Mk to Mkv models dropped the mean clock rate

316 estimate by about $1 / 3$ in both the autapomorphies-included and autapomorphies-excluded

317 dataset. This would affect the morphological branchlengths (number of expected changes per

318 site) in a similar way. As $\mathrm{M} k_{\text {parsinf }}$ includes the $\mathrm{Mkv}$ correction, this suggests $\mathrm{M} k_{\text {parsinf }}$ would have

319 the same or greater effect.

320

321 Two comments we received from readers of a draft of this manuscript deserve attention. First,

322 Mike Lee (personal communication) pointed out that the equations above would be somewhat

323 different if the researchers coding characters excluded not only parsimony-uninformative

324 characters, but also characters that were "partially uninformative." An example would be the

325 character pattern 00112 - character states 0 and 1 are potential synapomorphies, but character

326 state 2 is an autapomorphy. Above, we have focused on the "literal" interpretation of

327 "parsimony-informative," which we think is the understanding commonly used in the literature

328 and in programs. We suggest that an ascertainment bias correction that assumes the

329 unobservability of invariant, parsimony-uninformative, and partially-parsimony-informative

330 characters should have a new name, perhaps simply "partial-parsinf." 
332 Second, Mark Holder (personal communication) pointed out that the scalability problem is less

333 detrimental, although still daunting, if it is realized that some patterns will have the same

334 likelihood under the $\mathrm{Mk}$ model (because it is a symmetric-equal-rates model). For example, the 335 patterns 00112, 00221, 11002, 11220, 22110, and 22001, will all have the same likelihood.

336 Therefore the log-likelihood can be calculated for one of these patterns, and multiplied by the

337 number of patterns in that category. This amounts to removing $i$ ! from equation (5), and using

338 it as a weight to multiply by the log-likelihood of a pattern. Beast2 does have a "weight" option

339 for its Alignment class, but we have not tested it in combination with the

340 ascertained/excludefrom/excludeto options in the XML.

341

342 Equation (5) applies to unordered characters, where any autapomorphies will be parsimony-

343 uninformative. If it is instead assumed that the characters are ordered, then any pattern with

344 more than two states will be parsimony-informative. For example, the pattern 011112 would

345 be parsimony-uninformative for an unordered character, but parsimony-informative for an

346 ordered character, because bipartitions grouping states $(0,1)$ and $(1,2)$ would be favoured over

347 trees grouping $(0,2)$. Thus, the number of unobservable patterns (assuming the researchers

348 doing the character scoring had this in mind when building their matrix) is much reduced, since 349 only patterns with 1 or 2 character states are unobservable. The equation is:

$$
\sum_{i=1}^{2}\left(\begin{array}{l}
k \\
i
\end{array}\right)\left(\begin{array}{l}
i \\
1
\end{array}\right)\left(\begin{array}{c}
n \\
i-1
\end{array}\right)(i-1) !
$$


351

352 The unobservable pattern counts for an ordered character are shown in Supplemental Table 4, 353 and fractions in Supplemental Table 5.

354

355 Resolution of the discussion about when and where $\mathrm{M} k_{\text {parsinf }}$ is functional, useful, or 356 unnecessary may be difficult, as it depends in part on gnarly philosophical questions about what the "complete" morphology matrix would look like (how many invariant morphological characters are "truly" observable in any particular clade?). This is closely tied to another difficult question: what is the "true" morphological rate for "all" of the morphology? We can briefly suggest that probably such questions are almost unanswerable in the abstract, and that any meaningful statements about rates and completeness must be made with reference to some method of character collection. It certainly appears that these problems should be studied more carefully than can be done here. Unless these issues are resolved, however, it may be that including all codeable autapomorphies, and using the Mkv ascertainment bias correction, is the best option.

\section{Data accessibility}

369 All data, code, and Beast2 inputs and outputs are available in this article's Dryad data repository: http://datadryad.org/review?doi=doi:10.5061/dryad.8q4c8 
373

\section{Acknowledgements}

375 We would like to thank April Wright (ORCID ID: 0000-0003-4692-3225), David Bapst (ORCID ID:

376 0000-0002-9087-1103), Graeme Lloyd (ORCID ID: 0000-0001-6887-3981), Daniel Ksepka, James

377 Parham (ORCID ID: 0000-0002-5221-0072), Nathan Smith, Alan Turner, and Mike Lee for helpful

378 discussions, and especially helpful comments from Mark Holder (ORCID ID: 0000-0001-5575-

379 0536) and two other reviewers.

380 
381

382

383

384

385

386

387

388

389

390

391

392

393

394

395

396

397

398

399

400

401

402

403

404

405

406

407

408

409

410

411

412

413

414

415

416

417

418

419

420

421

422

423

\section{References}

Alexandrou MA, Swartz BA, Matzke NJ, and Oakley TH. 2013. Genome duplication and multiple evolutionary origins of complex migratory behavior in Salmonidae. Molecular Phylogenetics and Evolution 69:514-523. 10.1016/j.ympev.2013.07.026

Allman ES, Holder MT, and Rhodes JA. 2010. Estimating trees from filtered data: Identifiability of models for morphological phylogenetics. Journal of Theoretical Biology 263:108-119. 10.1016/j.jtbi.2009.12.001

Bouckaert R, Heled J, Kühnert D, Vaughan T, Wu C-H, Xie D, Suchard MA, Rambaut A, and Drummond AJ. 2014. BEAST 2: a software platform for Bayesian evolutionary analysis. PLoS Comput Biol 10:e1003537. 10.1371/journal.pcbi.1003537

Dembo M, Radovčić D, Garvin HM, Laird MF, Schroeder L, Scott JE, Brophy J, Ackermann RR, Musiba CM, de Ruiter DJ, Mooers $A \varnothing$, and Collard M. 2016. The evolutionary relationships and age of Homo naledi: An assessment using dated Bayesian phylogenetic methods. Journal of Human Evolution 97:17-26. http://dx.doi.org/10.1016/j.jhevol.2016.04.008

dos Reis M, Donoghue PCJ, and Yang Z. 2016. Bayesian molecular clock dating of species divergences in the genomics era. Nature Reviews: Genetics 17:71-80. 10.1038/nrg.2015.8

Drummond AJ, and Bouckaert RR. 2015. Bayesian Evolutionary Analysis with BEAST: Cambridge University Press.

Felsenstein J. 1992. Phylogenies from restriction sites: a maximum-likelihood approach. Evolution 46:159-173. 10.2307/2409811

Gavryushkina A, Heath TA, Ksepka DT, Stadler T, Welch D, and Drummond AJ. 2015. Bayesian total evidence dating reveals the recent crown radiation of penguins. arXiv. arXiv:1506.04797

Gavryushkina A, Welch D, Stadler T, and Drummond AJ. 2014. Bayesian inference of sampled ancestor trees for epidemiology and fossil calibration. PLoS Comput Biol 10:e1003919. 10.1371/journal.pcbi.1003919

Goloboff PA, Farris JS, and Nixon KC. 2008. TNT, a free program for phylogenetic analysis. Cladistics 24:774-786. 10.1111/j.1096-0031.2008.00217.x

Hennig W, Davis DD, and Zangerl R. 1999. Phylogenetic Systematics: University of Illinois Press. Koch JM, and Holder MT. 2012. An Algorithm for Calculating the Probability of Classes of Data Patterns on a Genealogy. PLOS Currents Tree of Life. 10.1371/4fd1286980c08

Lee Michael SY, and Palci A. 2015. Morphological phylogenetics in the genomic age. Current Biology 25:R922-R929. 10.1016/j.cub.2015.07.009

Lewis PO. 2001. A likelihood approach to estimating phylogeny from discrete morphological character data. Systematic Biology 50:913-925. 10.1080/106351501753462876

Matzke NJ. 2015 BEASTmasteR: automated conversion of NEXUS data to BEAST2 XML format, for fossil tip-dating and other uses. PhyloWiki. http://phylo.wikidot.com/beastmaster

Matzke NJ. 2016. The evolution of antievolution policies after Kitzmiller versus Dover. Science 351:28-30. 10.1126/science.aad4057

Matzke NJ, and Wright AM. 2016. Inferring node dates from tip dates in fossil Canidae: the importance of tree priors. Biology Letters 12:20160328. 10.1098/rsbl.2016.0328 
424 Mishler BD. 2005. The logic of the data matrix in phylogenetic analysis. In: Albert VA, ed.

425

426

427

428

429

430

431

432

433

434

435

436

437

438

439

440

441

442

443

444

445

446

447

448

449

450

451

452

453

454

455

456

457

458

459

460

461
Parsimony, phylogeny, and genomics. Oxford: Oxford University Press, 57-70.

Müller J, and Reisz RR. 2006. The phylogeny of early eureptiles: comparing parsimony and Bayesian approaches in the investigation of a basal fossil clade. Systematic Biology 55:503-511. 10.1080/10635150600755396

Nylander JAA, Ronquist F, Huelsenbeck JP, and Nieves-Aldrey J. 2004. Bayesian phylogenetic analysis of combined data. Systematic Biology 53:47-67. 10.1080/10635150490264699

Parham JF, Donoghue PCJ, Bell CJ, Calway TD, Head JJ, Holroyd PA, Inoue JG, Irmis RB, Joyce WG, Ksepka DT, Patané JSL, Smith ND, Tarver JE, van Tuinen M, Yang Z, Angielczyk KD, Greenwood JM, Hipsley CA, Jacobs L, Makovicky PJ, Müller J, Smith KT, Theodor JM, Warnock RCM, and Benton MJ. 2012. Best practices for justifying fossil calibrations. Systematic Biology 61:346-359. 10.1093/sysbio/syr107

Pyron RA. 2011. Divergence Time Estimation Using Fossils as Terminal Taxa and the Origins of Lissamphibia. Systematic Biology 60:466-481. 10.1093/sysbio/syr047

Rambaut A, Lam TT, Max Carvalho L, and Pybus OG. 2016. Exploring the temporal structure of heterochronous sequences using TempEst (formerly Path-O-Gen). Virus Evolution 2:vew007-vew007. 10.1093/ve/vew007

Ronquist F, Huelsenbeck J, and Teslenko M. 2011 Draft MrBayes version 3.2 manual: tutorials and model summaries. SourceForge. http://mrbayes.sourceforge.net/mb3.2 manual.pdf

Ronquist F, and Huelsenbeck JP. 2003. MrBayes 3: Bayesian phylogenetic inference under mixed models. Bioinformatics 19:1572-1574. 10.1093/bioinformatics/btg180

Ronquist F, Klopfstein S, Vilhelmsen L, Schulmeister S, Murray DL, and Rasnitsyn AP. 2012. A total-evidence approach to dating with fossils, applied to the early radiation of the Hymenoptera. Systematic Biology 61:973-999. 10.1093/sysbio/sys058

Stadler T. 2015 TreeSim: Simulating Phylogenetic Trees. https://CRAN.Rproject.org/package=TreeSim

Swofford DL. 2003 PAUP*. Phylogenetic Analysis Using Parsimony, version 4. http://paup.csit.fsu.edu/

Wood HM, Matzke NJ, Gillespie RG, and Griswold CE. 2013. Treating fossils as terminal taxa in divergence time estimation reveals ancient vicariance patterns in the Palpimanoid spiders. Systematic Biology 62:264-284. 10.1093/sysbio/sys092

Wright AM, and Hillis DM. 2014. Bayesian analysis using a simple likelihood model outperforms parsimony for estimation of phylogeny from discrete morphological data. PLOS ONE 9:e109210. 10.1371/journal.pone.0109210

Yeates D. 1992. Why remove autapomorphies? Cladistics 8:387-389. 10.1111/j.1096-

0031.1992.tb00080.x 
Figure 1 (on next page)

Comparison of the tip-dated phylogenies of early eureptiles inferred when excluding (A) or including (B) autapomorphies, under Mkv ascertainment bias correction.

Numbers are posterior probabilities. Bars represent the 95\% HPD. 
A. Mkv, autapomorphies excluded

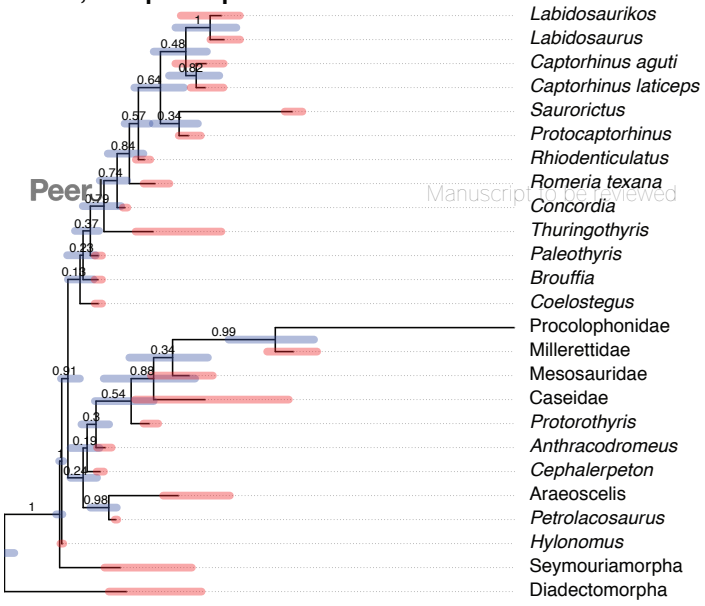

B. Mkv, autapomorphies included

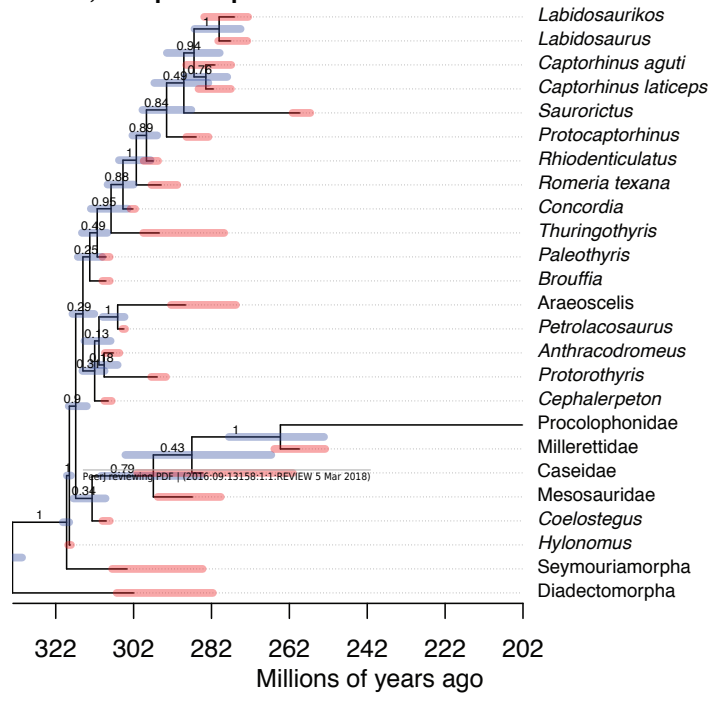


Figure 2 (on next page)

Simulation procedure and results.

Simulation procedure (A) and results (B-E). The lack of an effect of excluding autapomorphies on dating in the empirical eureptile result is similar to the result on non-clock data shown in $2 \mathrm{E}$. 
Character datasets:

Beast2 estimation:

Conclusion:

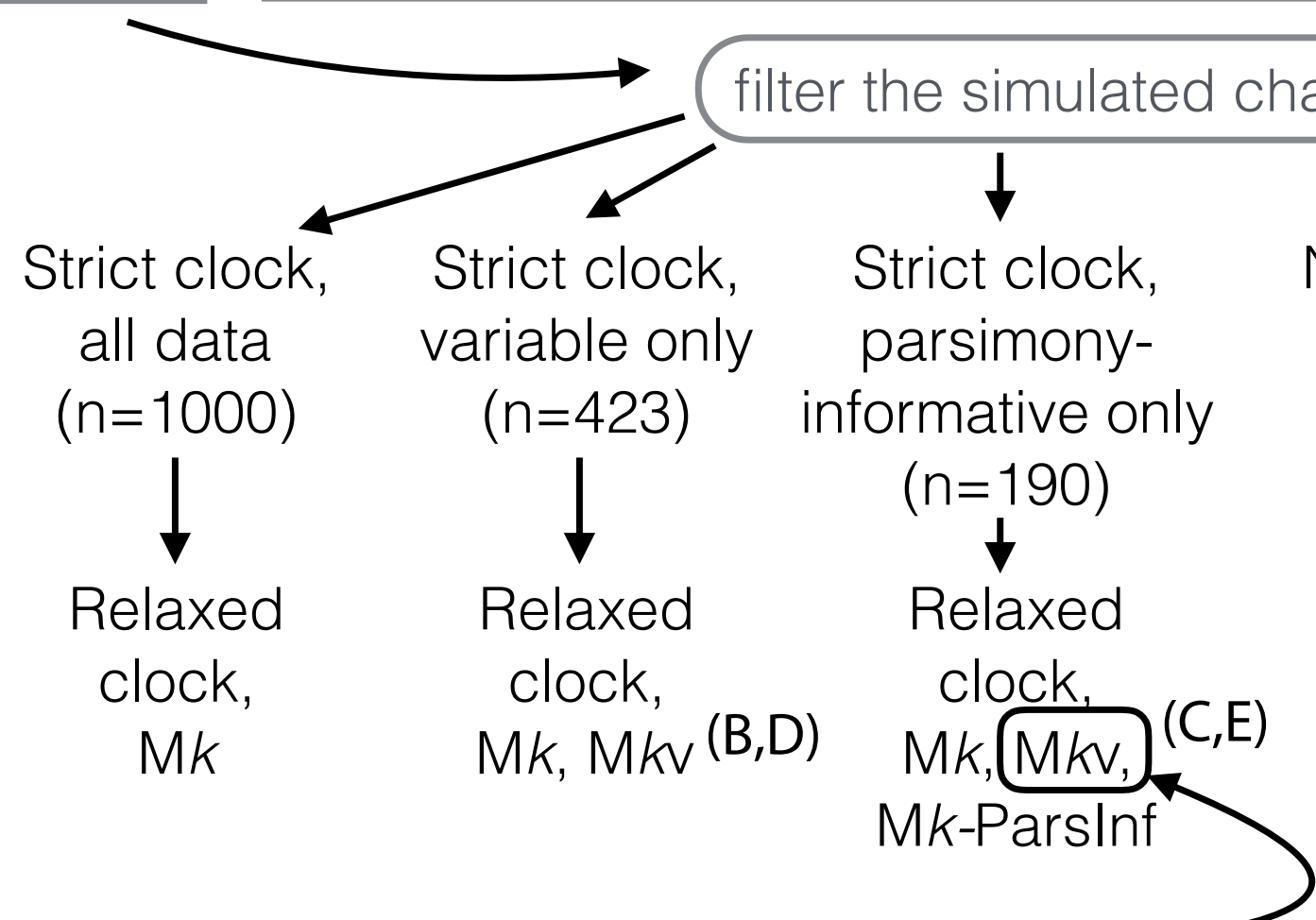

Strong bias towards underestimating terminal branch lengths is observed, as predicted by theory

B.
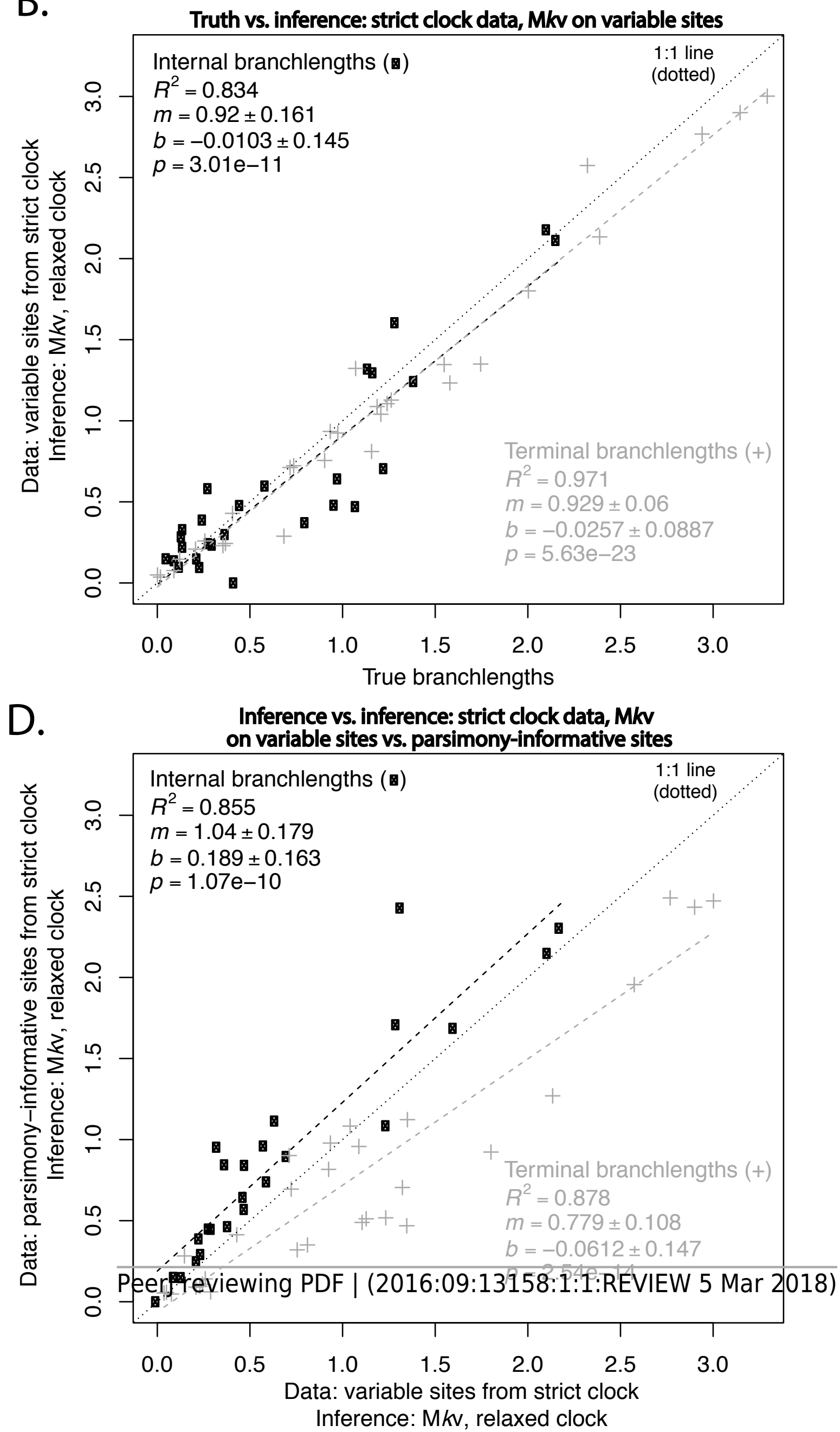

C.

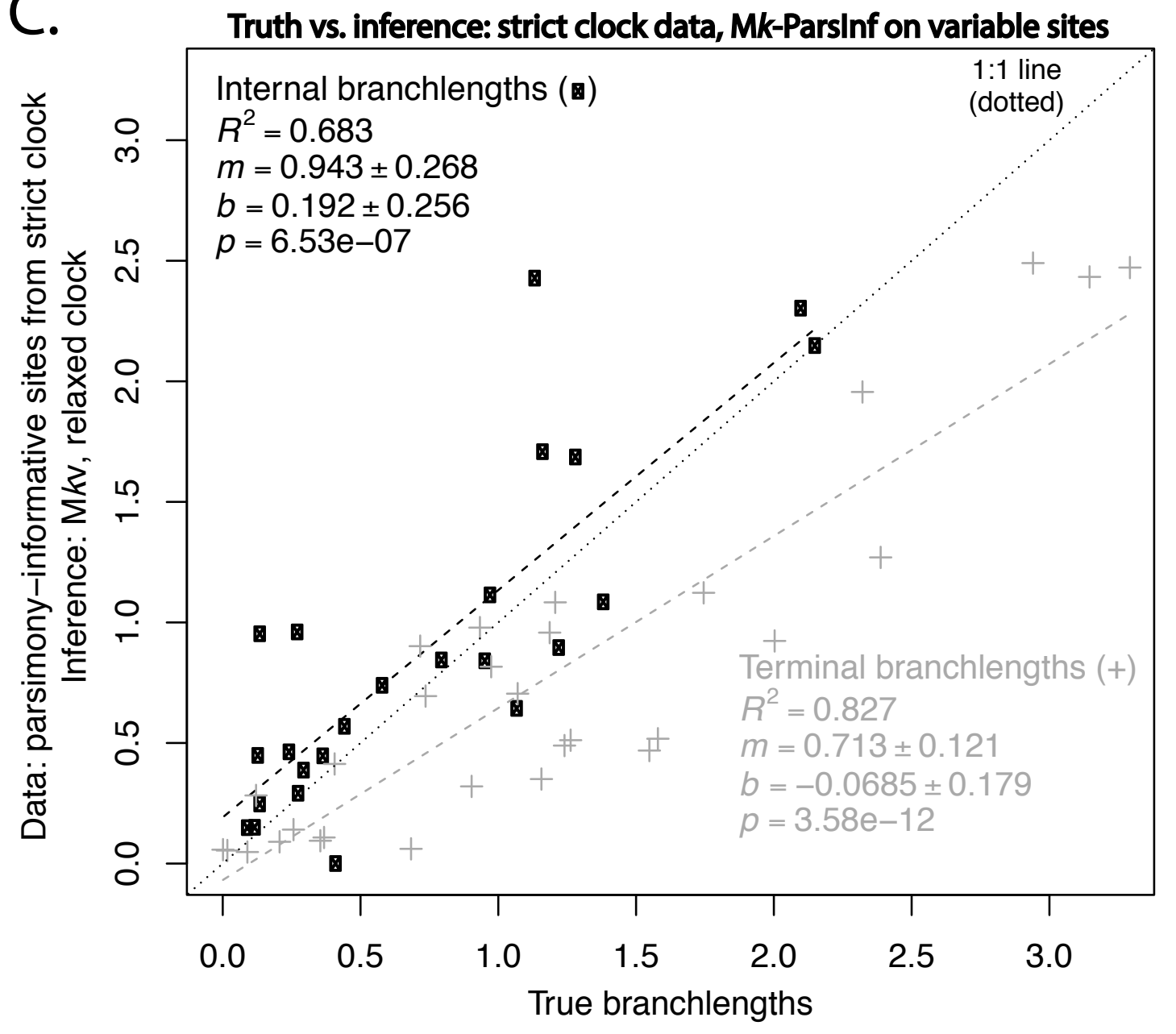

E.

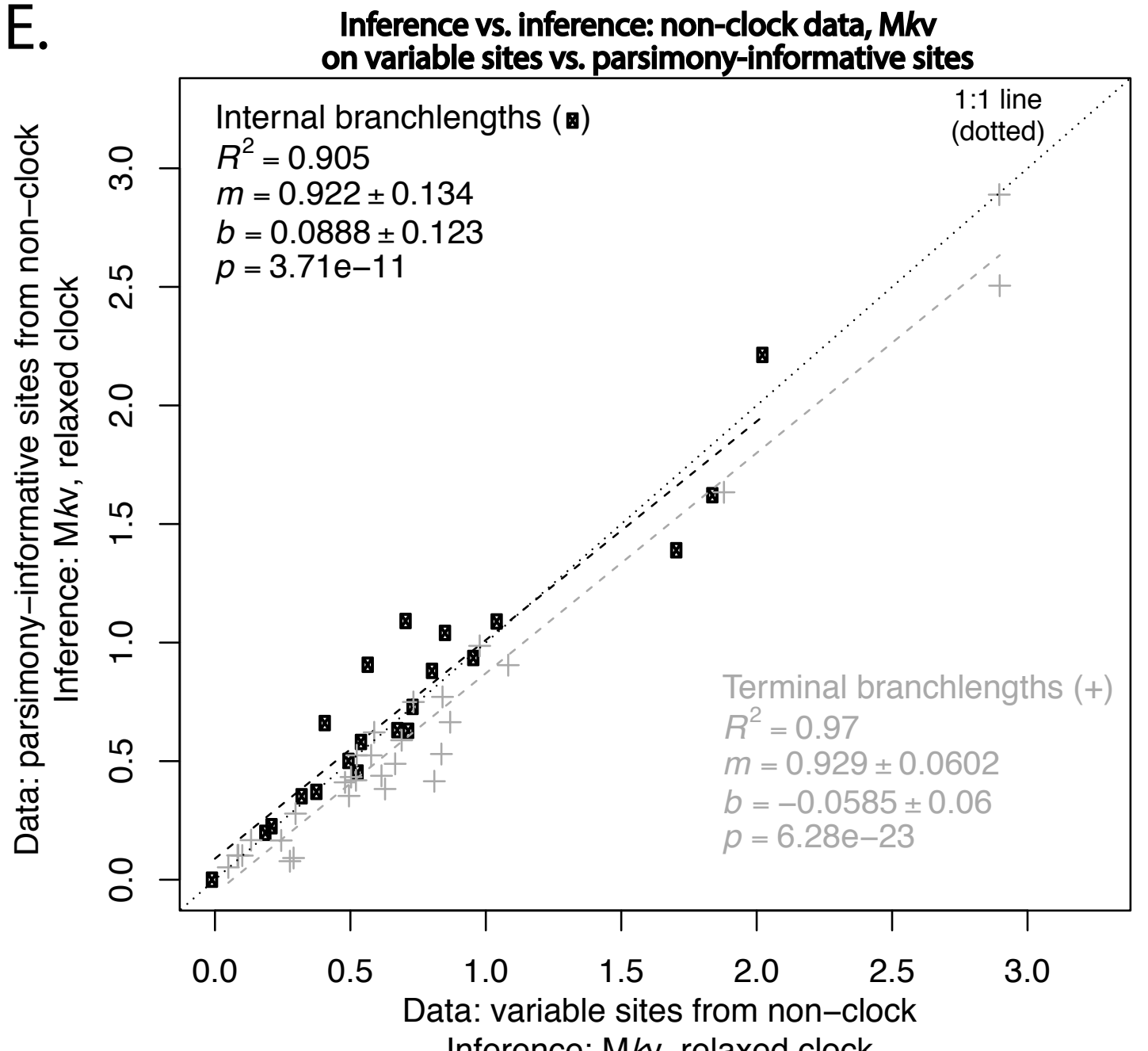

No strong bias towards underestimating terminal branch lengths is observed, because characters are not clocklike, so estimated rates and branch lengths have high variance (similar to Müller/Reisz dataset) clock, Mk

all data

$(n=1000)$<smiles>[3H][3H]</smiles>

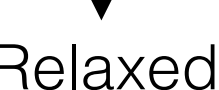

Relaxed clock, (D)
Non-clock, parsimonyinformative only<smiles>[AsH2][AsH2][AsH2]</smiles>

Relaxed clock $\mathrm{Mk}$ Mkv Mk-Parsinf<smiles>C1CCCCC1</smiles> 


\section{Table $\mathbf{1}$ (on next page)}

Comparison of summary statistics from the five Beast2 runs using "best-practices" tip dates. 


\begin{tabular}{|c|c|c|c|c|c|}
\hline Run \# & 1 & 2 & 3 & 4 & 5 \\
\hline Data & \multicolumn{2}{|c|}{ Including autapomorphies } & \multicolumn{3}{|c|}{ Excluding autapomorphies } \\
\hline Model & $\mathrm{M} k$ & $\mathrm{Mkv}$ & $\mathrm{M} k$ & $\mathrm{M} k \mathrm{v}$ & $\mathrm{M} k$-parsinf \\
\hline Ln Posterior & -1393.4 & -1362.2 & -1154.2 & -1144.9 & -1134.4 \\
\hline ESS & 1801 & 1485 & 1801 & 1801 & 1801 \\
\hline Root age & $332.6[330.2,335.3]$ & $332.5[330.0,335.1]$ & $\begin{array}{l}332.6[330.1, \\
335.1]\end{array}$ & $332.6[330.1,335.1]$ & $\begin{array}{l}332.6[330.0, \\
335.1]\end{array}$ \\
\hline Birth & $\begin{array}{l}0.360[0.0355, \\
1.316]\end{array}$ & $\begin{array}{l}0.424[0.0405, \\
1.708]\end{array}$ & $\begin{array}{l}0.342[0.0463, \\
1.221]\end{array}$ & $\begin{array}{l}0.381[0.0402, \\
1.377]\end{array}$ & $\begin{array}{l}0.564[0.0444, \\
2.841]\end{array}$ \\
\hline Death & $\begin{array}{l}0.336 \text { [9.17e-5, } \\
1.315]\end{array}$ & $\begin{array}{l}0.3995[1.13 \mathrm{e}-4 \\
1.723]\end{array}$ & $\begin{array}{l}0.318 \text { [4.97e-6, } \\
1.220]\end{array}$ & $\begin{array}{l}0.357[2.57 \mathrm{e}-4, \\
1.391]\end{array}$ & $\begin{array}{l}0.541[6.37 \mathrm{e}-4, \\
2.843]\end{array}$ \\
\hline Sampling & $\begin{array}{l}0.0271[7.90 \mathrm{e}-4, \\
0.0626]\end{array}$ & $\begin{array}{l}0.0264[0.00104 \\
0.0650]\end{array}$ & $\begin{array}{l}0.0271[8.85 \mathrm{e}-4, \\
0.063]\end{array}$ & $\begin{array}{l}0.0261[9.96 \mathrm{e}-4, \\
0.0634]\end{array}$ & $\begin{array}{l}0.0256[7.66 \mathrm{e}-4, \\
0.0643]\end{array}$ \\
\hline $\begin{array}{l}\text { Clock rate } \\
\text { mean }\end{array}$ & $\begin{array}{l}0.0782[0.015 \\
0.159]\end{array}$ & $\begin{array}{l}0.0376[0.0074 \\
0.0840]\end{array}$ & $\begin{array}{l}0.788 \text { [0.0305, } \\
3.982]\end{array}$ & $\begin{array}{l}0.550[0.0228, \\
2.655]\end{array}$ & $\begin{array}{l}0.235[0.0142, \\
0.664]\end{array}$ \\
\hline $\begin{array}{l}\text { Clock rate } \\
\text { SD }\end{array}$ & $1.747[1.201,2.399]$ & $1.712[1.111,2.309]$ & $\begin{array}{l}2.436[1.572, \\
3.477]\end{array}$ & $2.341[1.488,3.379]$ & $\begin{array}{l}2.079[1.318, \\
2.984]\end{array}$ \\
\hline
\end{tabular}




\section{Table 2 (on next page)}

Number of patterns that are unobservable in the $M k_{\text {parsinf }}$ model. 


\begin{tabular}{|l} 
Table 2. Number of patterns that are unobservable in the M $k_{\text {parsinf }}$ model. \\
\hline \\
\hline
\end{tabular}

1 